\title{
Improving image contrast using coded excitation for ultrasonic imaging
}

\author{
Jose R. Sanchez \\ Electrical and Computer Engineering Department \\ Bradley University \\ Peoria, Illinois 61525 \\ Email: jsm@bradley.edu
}

\author{
Marko Orescanin and Michael Oelze \\ Electrical and Computer Engineering Department \\ University of Illinois \\ Urbana, Illinois 61801 \\ Email: oelze@illinois.edu
}

\begin{abstract}
A method for improving the contrast resolution while maintaining the $-6 \mathrm{~dB}$ axial resolution of ultrasonic Bmode images is proposed. The technique proposed is known as eREC-FC, which enhances a recently developed REC-FC technique. REC-FC is a combination of the coded excitation technique known as resolution enhancement compression (REC) and the speckle-reduction technique frequency compounding (FC). In REC-FC, the speckle is reduced and the contrast is improved but at the expense of a reduction in axial resolution. However, by compounding various REC-FC images made from various subband widths the tradeoff between axial resolution and contrast enhancement can be extended. Simulations and experimental measurements were conducted with a single-element transducer (f/2.66) having a center frequency of $2.25 \mathrm{MHz}$ and a -3-dB bandwidth of $50 \%$. Measurements of tissue-mimicking targets of various contrast levels and diameters were imaged. The image quality metrics used to compare eREC-FC to conventional pulsing (CP), REC, and REC-FC were contrast-to-noise, and histogram overlap. Maximum and minimum increases in CNR of $189 \%$ and $88 \%$ were observed when comparing eREC-FC to CP for the $12 \mathrm{~mm}$ in diameter $+6 \mathrm{~dB}$ and $-3 \mathrm{~dB}$ targets, respectively. Image histrogram analysis between the target and background regions suggested that improved lesion detectability was observed by using eREC-FC as the overlap between the two histograms was minimized. With eREC-FC technique the axial resolution was preserved (i.e., same axial resolution that would be typically observed with conventional excitation with a pulse).
\end{abstract}

\section{INTRODUCTION}

In imaging, the ability to detect small or low-contrast structures is of utmost importance. However, ultrasonic images are riddled with speckle, which reduces the ability to detect low-contrast targets. Speckle is formed by subresolution scatterers that cause constructive and destructive interference of backscattered ultrasonic signals within the resolution cell volume of an ultrasonic source [1]. Consequently, speckle reduction techniques must be applied to improve image contrast and enhance the detectability of structures having low contrast with the background [2].

A recently developed speckle reduction techniqueresolution enhancement compression-frequency compounding (REC-FC) - improves the contrast of ultrasonic images [3]. REC-FC used the coded excitation and pulse compression technique, REC, which improves the axial resolution of an ultrasonic imaging system by a factor of 2 [4]. A larger axial resolution translated into a larger bandwidth. This larger bandwidth was exploited by combining the REC technique with FC. FC is a speckle reduction technique that subdivides the spectrum of the radio-frequency (RF) echoes into subbands to make partially uncorrelated images [5]. These images were then compounded to reduce the speckle variance. REC-FC was found to improve image contrast by as much as $231 \%$ compared to a conventional pulsing (CP) scheme. Overall, RECFC improved image quality, contrast and lesion boundaries. However, the drawback of REC-FC was that subbands only contained a fraction of the original system bandwidth, which resulted in a reduction of axial resolution. In this study, an enhanced REC-FC technique (eREC-FC) is proposed, which enhances the contrast of an ultrasonic image without the same loss in axial resolution.

\section{Problem Formulation}

In REC, a pre-enhanced chirp, $x(t)$, is used to excite an ultrasonic focused source, $h(t)$, as shown in Fig. 1. The preenhanced chirp is obtained through convolution equivalence as discussed in [3], [4]. The goal of the pre-enhanced chirp is to boost the energy in the band edges of the source's frequency response. $h(t)$ corresponds to the pulse-echo impulse response of an ultrasonic source at its focal length. Upon excitation of the source, a pressure waveform propagates through the medium. A fraction of this pressure is reflected from scatterers located in the field of view of the source. The reflection of a single point scatterer (echo) at the focus is shown as $y(t)$ in Fig. 1.

With REC, the spectral support of the echo signal is much larger than the bandwidth of the source. However, the pulse duration of $y(t)$ is longer than an echo from the same source when the excitation is $\delta(t)$, which will be described as conventional pulsing $(\mathrm{CP})$ in this study. Therefore, in order to recover the benefits of the larger bandwidth, the resolution must be restored through pulse compression. Pulse compression is performed using a Wiener filter, which is described by [6]:

$$
H_{\text {Wiener }}=\frac{\Psi^{*}(f)}{|\Psi(f)|^{2}+\gamma \overline{e S N R(f)}},
$$

where $\gamma$ is a smoothing parameter that controls the tradeoff between sidelobe levels, axial resolution, and echo signalto-noise ratio $(e S N R)$ [7]. $\overline{e S N R}$ is the echo signal-to- 

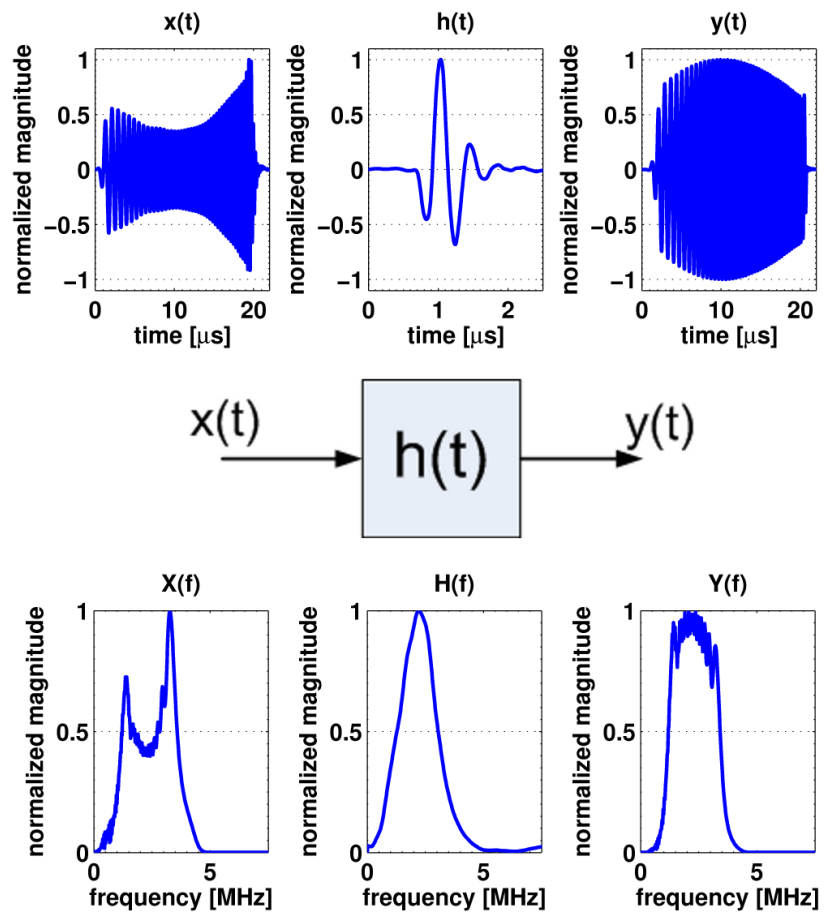

Fig. 1. Excitation of an ultrasound system, $h(t)$ by a pre-enhanced chirp, $x(t)$, and its resulting output, $y(t) . X(f), H(f)$, and $Y(f)$ correspond to the Fourier transform of $x(t), h(t)$, and $y(t)$, respectively.
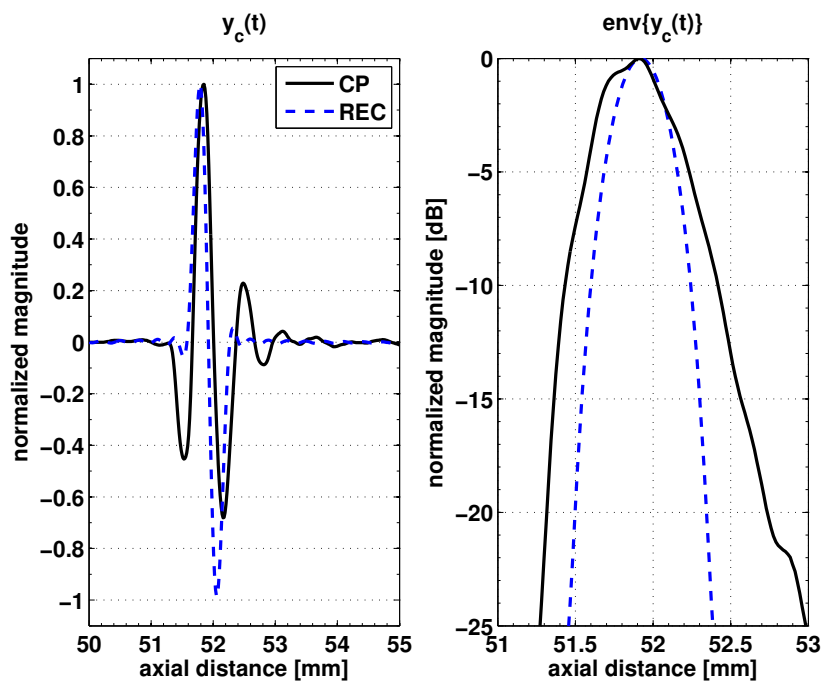

Fig. 2. Compression of the $y(t)$ is represented by $y_{c}(t)$ and the logcompressed and envelope detected version of $y_{c}(t)$.

noise ratio per frequency channel and $\Psi(f)$ corresponds to the Fourier transform of a linear chirp excitation, which is part of convolution equivalence scheme used to obtain the pre-enhanced chirp as discussed in [3], [4]. The compressed echo waveform and the log-compressed envelope of the echo waveform for a point target are shown in Fig. 2 along with the $\mathrm{CP}$ reference.

In REC-FC, the wideband RF spectrum of each scan line
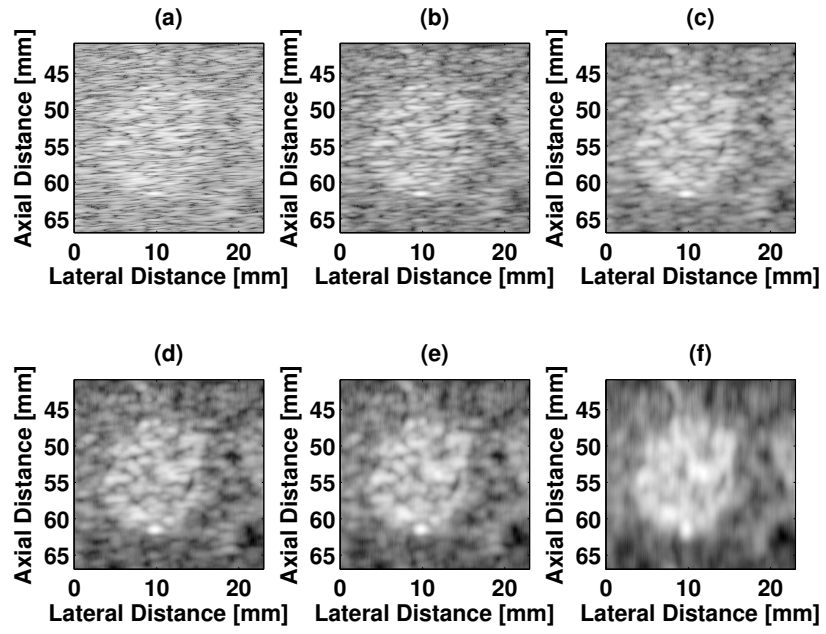

Fig. 3. B-mode images for (a) REC reference, (b) REC-FC (full width), (c) REC-FC (half width), (d) REC-FC (third-width), (e) REC-FC (fourth-width), REC-FC (eight-width). Image dynamic range is $-50 \mathrm{~dB}$.

was partitioned into $N$ subbands by using Guassian band-pass filters. These Gaussian band-pass filters contained a fraction of the original system bandwidth. The resulting images from the $N$ subbands were compounded to form an image with reduced speckle variance. A reduction in speckle variance translated into improvement of contrast. However, because the subband width was smaller in bandwidth than the original system, the axial resolution in the compounded images deteriorated. The tradeoff of axial resolution vs. image contrast is shown in Fig. 3.

In this study, a method is proposed that could provide the improvements in contrast obtained with REC-FC without degrading the $-6 \mathrm{~dB}$ axial resolution beyond the axial resolution obtained for $\mathrm{CP}$. The proposed method consists of compounding REC-FC images obtained from different subband widths, which shall reduce the speckle variance even further and result in an improvement of image contrast. This technique shall be known as enhanced REC-FC, or eREC-FC. In this study, eREC-FC utilized the uniformly weighted sum of the following images (Fig. 3): REC reference image, RECFC (full width), REC-FC (half width), REC-FC (third-width), REC-FC (fourth-width), and REC-FC (eighth-width) to form an enhanced final REC-FC image.

Theoretically, by summing all the compounded images along with the reference, the final enhanced image would have a $-6 \mathrm{~dB}$ axial resolution similar to the full width REC-FC scenario or equivalent to the original resolution obtained with $\mathrm{CP}$. The results of summing the envelope of the reference and subbands are shown in Fig. 4. Evaluation of the eREC-FC envelope at $-6 \mathrm{~dB}$ in Fig. 4(b) indicated that a loss of 10 $\mu \mathrm{m}$ in axial resolution was obtained when compared to $\mathrm{CP}$. Furthermore, every drop of $-6 \mathrm{~dB}$ in amplitude is followed by a slight deterioration in the axial resolution. However, this degradation should not affect the image quality unless there is a large contrast difference, such as in a cystic lesion (i.e., no 


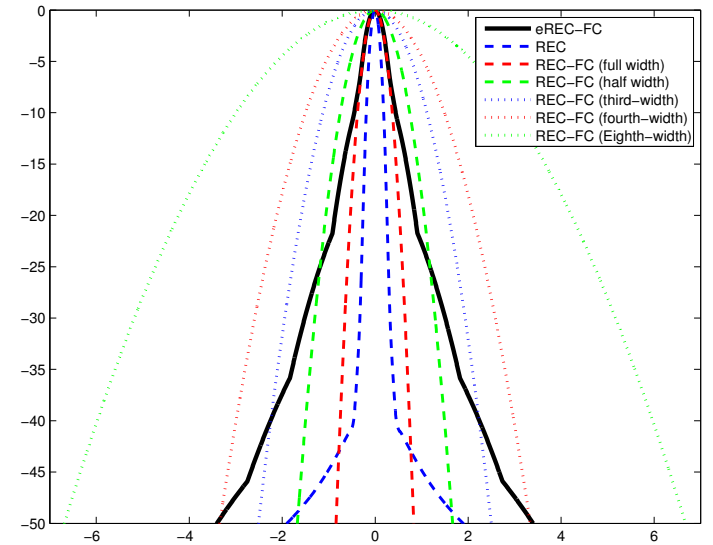

(a)

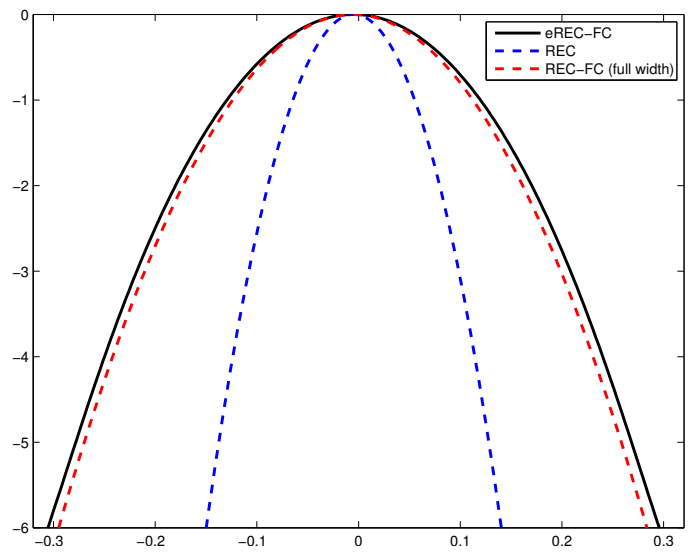

(b)

Fig. 4. (a) Individual envelopes showcasing the axial resolution for the REC reference case , the REC-FC cases, and the eREC-FC case(b) zoomed version of eREC-FC showing that the axial resolution was similar to REC-FC (full width). Note that the axial resolution for REC-FC (full width) is the same for CP.

scatterers).

1) Experimental setup: Experimental measurements of physical phantoms were performed with a single-element, weakly focused (f/2.66) transducer (Panametrics, Waltham, MA) with a nominal center frequency of $2.25 \mathrm{MHz}$ and a $50 \%$ (at $-3 \mathrm{~dB}$ pulse-echo) fractional bandwidth (measured). The phantom was imaged by translating the transducer laterally across the phantom. There were two different experimental setups utilized: one for $\mathrm{CP}$ methods and another one for REC experiments. These setups would contain different noise levels due to the use of different excitation systems. Therefore, to avoid errors in the comparisons, the noise levels were normalized to an eSNR of $28 \mathrm{~dB}$. Normalization of eSNR was accomplished by adding zero-mean Gaussian white noise to the CP RF echo waveform. The two experimental setups are described as follows:

1) Conventional pulsing experimental setup: The transducer was excited by a pulser-receiver (Panametrics 5800, Waltham, MA) and the receive waveform was displayed on an oscilloscope (Lecroy 9354 TM, Chester
Ridge, NY) for visual verification. The echo signal was recorded at a rate of $100 \mathrm{MHz}$ by a 12-bit A/D (Strategic Test Digitizing Board UF3025, Cambridge, MA) for further processing by a PC.

2) REC experimental setup: The pre-enhanced chirp was generated in MATLAB (The Mathworks Inc., Natick, MA) and downloaded to an arbitrary waveform generator (Tabor Electronics W1281A, Tel Hanan, Israel). The excitation signal was sampled at a rate of 100 $\mathrm{MHz}$ and amplified by an RF power amplifier (ENI 3251, Rochester, NY). The amplified signal (50 dB) was connected to the transducer through a diplexer (Ritec RDX-6, Warwick, RI). The echo signal was received by a pulser-receiver (Panametrics 5800, Waltham, MA), which was displayed on an oscilloscope (Lecroy 9354 TM, Chester Ridge, NY) for visual verification. The echo signal was recorded at a rate of $100 \mathrm{MHz}$ by a 12-bit A/D (Strategic Test Digitizing Board UF3025, Cambridge, MA) for further processing by a PC.

A tissue-mimicking phantom (ATS Laboratories Model 539, Bridgeport, CT) and a hydrogel cone phantom were used to assess the performance of eREC-FC. The material from the ATS tissue-mimicking phantom consisted of urethane rubber with a speed of sound of $1450 \mathrm{~m} / \mathrm{s} \pm 1.0 \%$ at $23{ }^{\circ} \mathrm{C}$ and an attenuation coefficient of $0.5 \mathrm{~dB} \mathrm{MHz}^{-1} \mathrm{~cm}^{-1} \pm 5.0 \%$. Fifteen $\mathrm{mm}$ diameter echogenic grayscale target structures with contrasts of $+6 \mathrm{~dB},+3 \mathrm{~dB},-3 \mathrm{~dB}$, and $-6 \mathrm{~dB}$ located at a depth of $4 \mathrm{~cm}$ into the phantom were imaged. The hydrogel cone phantom was designed and fabricated at the Ultrasonic Imaging Laboratory at the Beckman Institute at the University of Illinois at Urbana-Champaign. The phantom contained a cone inclusion with a $15 \mathrm{~mm}$ diameter at the top. By imaging cross-sectional planes of the cone at different heights of the cone, lesions of different diameters could be imaged and evaluated. For the purpose of this study, lesion diameters were $8 \mathrm{~mm}, 5 \mathrm{~mm}$ and $3 \mathrm{~mm}$. The materials (and relative amounts by weight) for the cone inclusion were type-A gelatin (Fisher Scientific, Pittsburgh, PA) (5.5\%), n-propanol $(9.0 \%)$, distilled and deionized water $(83.4 \%)$, formaldehyde $(0.1 \%)$, and powdered graphite $(2.0 \%)$. The materials (and relative amounts by weight) for the surrounding medium around the cone were type-A gelatin (5.5\%), n-propanol $(9.0 \%)$, distilled and deionized water $(84.9 \%)$, formaldehyde $(0.1 \%)$, and powdered graphite $(0.5 \%)$. The powdered graphite scatterers were positioned at random locations throughout the phantom by rotating the phantom before the gel congealed. In addition, the concentration difference of the powdered graphite between the cone lesion and the background provided cone-to-background contrast of $+6 \mathrm{~dB}$. The speed of sound and the attenuation coefficient slope of the phantom were measured to be approximately equal to $1540 \mathrm{~m} / \mathrm{s}$ and $0.49 \mathrm{~dB} \mathrm{MHz}^{-1} \mathrm{~cm}^{-1}$, respectively, when using a $2.25 \mathrm{MHz}$ source. All measurements of phantoms were conducted at room temperature (approx. $23{ }^{\circ} \mathrm{C}$ ) in a tank of degassed water.

2) Image quality metrics: To evaluate the performance of the eREC-FC technique compared to CP, REC, and REC-FC 
the following image quality metrics were used:

1) Contrast-to-noise ratio (CNR): CNR [2], also known as contrast-to-speckle ratio, is a common quantitative measure that can assess image quality and describe the ability to perceive a target from the background region. CNR is defined as

$$
C N R=\left|\frac{\mu_{B}-\mu_{T}}{\sqrt{\sigma_{B}+\sigma_{T}}}\right|,
$$

where $\mu_{B}$ and $\mu_{T}$ are the mean intensity of the background and the target lesion, and $\sigma_{B}^{2}$ and $\sigma_{T}^{2}$ are the variance of the background and target, respectively. To avoid possible errors in the calculations due to attenuation, the evaluated regions of interest in the background and the target lesion were the same size and were located at the same depth.

2) Margin strength (MS): Estimates of MS [8] were used to detect the edges in the B-mode images. First, a $6 \mathrm{~dB}$ thresholding scheme was applied to the images. Then, MS was estimated to detect the strength of the boundaries using the following expression:

$$
M S=E\left\{\sqrt{\left(\frac{d R O I}{d x}\right)^{2}+\left(\frac{d R O I}{d y}\right)^{2}}\right\},
$$

where $E$ is the expectation operator, $R O I$ is the regionof-interest within the envelope and $x$ and $y$ corresponding to the image coordinates.

3) Histogram pixel intensity (HPI): HPI is the mean of the frequency distribution of grayscale pixel intensities and is described by

$$
H P I=E\{B\} .
$$

$B$ is the histogram being evaluated and is described by

$$
B(i)=c_{i},
$$

where $c_{i}$ represents the number of pixels in the image within a particular intensity level, $i$, which is an integer between 0 to 255 representing the grayscale levels used in B-mode images. Histograms were made for samesized regions for the target lesion and the background and located at the same depth. Ideally, for superior target detectability, there is no overlap present between the target histogram and the background histogram. Therefore, histogram overlap ( $\mathrm{HO})$, which is the percentage of overlapping pixels between these two regions was quantified.

\section{RESUlTS AND DISCUSSION}

The reference CP, refrence REC, REC-FC and eREC-FC Bmode images for the $+6 \mathrm{~dB}$ and $-3 \mathrm{~dB}$ ATS phantom targets are shown in Fig. 5-6, respectively. The CNR for the Bmode images for all the ATS phantom targets are listed in Table I. Examination of the B-mode images in Fig. 5 and Fig. 6 suggests that the spatial resolution of the eREC-FC image was similar to CP. Similar results were observed for the (a)

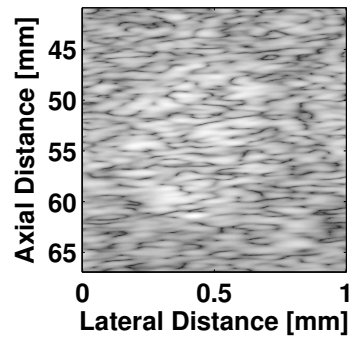

(c)

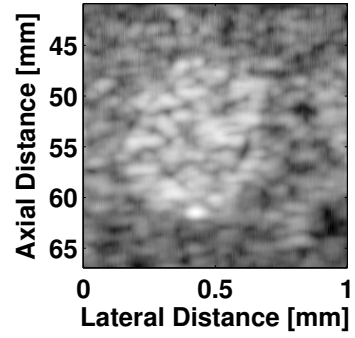

(b)

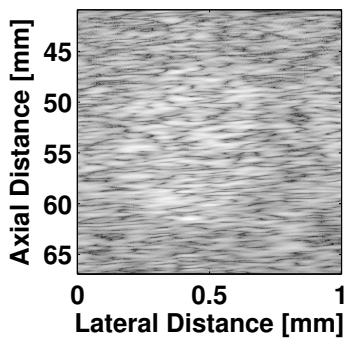

(d)

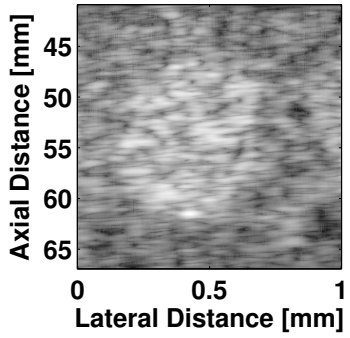

Fig. 5. B-mode images for the ATS phantom $12 \mathrm{~mm},+6 \mathrm{~dB}$ contrast target for (a) CP (reference), (b) REC (reference), (c) REC-FC (third-width), and (d) eREC-FC

$+3 \mathrm{~dB}$ and $-6 \mathrm{~dB}$ ATS phantom contrast targets. In addition to the resolution benefits of eREC-FC, the contrast of the B-mode images were improved by $189 \%, 151 \%, 89 \%$, and $136 \%$ for the $+6 \mathrm{~dB},+3 \mathrm{~dB},-3 \mathrm{~dB}$ and $-6 \mathrm{~dB}$ contrast targets, respectively. An average difference in $\mathrm{CNR}$ of approximately $10 \%$ was obtained between eREC-FC and REC-FC (thirdwidth) for all ATS contrast targets. Therefore, the RECFC (third-width) image is the appropriate choice for visual comparison between the eREC-FC and the standard RECFC technique. In the third-width case of REC-FC, the Bmode images appear blurry although the speckle variance was reduced, while in the eREC-FC technique the B-mode images appeared crisp but with more speckle.

REC-FC is known to enhance the boundaries of the lesions as shown in [3]. However, in eREC-FC, because images with variable speckle sizes are being compounded it was observed that the transition between the target and the background were blurred. Applying thresholding at $-6 \mathrm{~dB}$ along with MS resulted in Fig. 7. From the MS results, it was observed that REC-FC (third-width) had a more pronounced boundary compared to eREC-FC. Consequently, the tradeoff in using eREC-FC is a degradation of the enhanced edges obtained with the REC-FC technique in order to gain a contrast while maintaining the same axial resolution as $\mathrm{CP}$.

The reference, REC-FC and eREC-FC B-mode images for the $+6 \mathrm{~dB} 5 \mathrm{~mm}$ hydrogel target are shown in Fig. 8. Examination of the eREC-FC and the reference B-mode images in Fig. 8 also suggested that the spatial resolution of the eRECFC image is similar to CP. The eREC-FC images of the $+6 \mathrm{~dB}$ hydrogel phantom improved image contrast by $143 \%, 121 \%$, and $136 \%$ for the $8 \mathrm{~mm}, 5 \mathrm{~mm}$, and $3 \mathrm{~mm}$ lesion diameters, respectively. The CNR for the hydrogel cone phantom obtained 
(a)

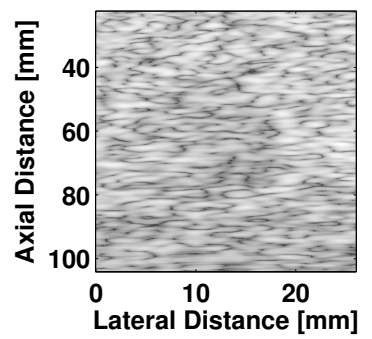

(c)

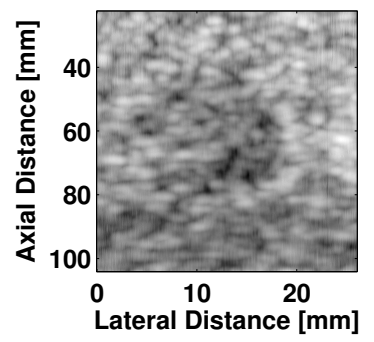

(b)

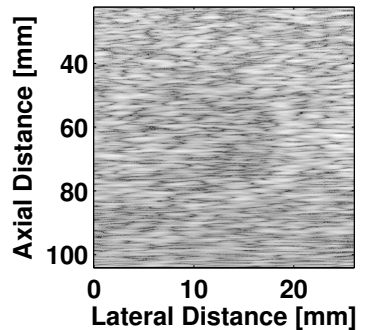

(d)

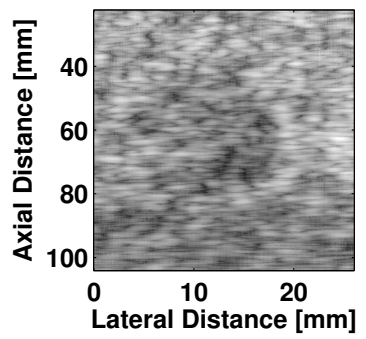

Fig. 6. B-mode images for the ATS phantom $12 \mathrm{~mm},-3 \mathrm{~dB}$ contrast target for (a) CP (reference), (b) REC (reference), (c) REC-FC (third-width), and (d) eREC-FC

TABLE I

CNR FOR VARIOUS CONTRAST TARGETS FOR CP, REC, REC-FC (THIRD-WIDTH) AND EREC-FC.

\begin{tabular}{c|cccc}
\hline \hline & \multicolumn{4}{|c}{ Technique $^{\mathrm{a}}$} \\
\cline { 2 - 5 } Lesion contrast/diameter & CP & REC & REC-FC & eREC-FC \\
\hline \hline$+6 \mathrm{~dB} / 12 \mathrm{~mm}$ & 0.39 & 0.46 & 1.39 & 1.13 \\
$+3 \mathrm{~dB} / 12 \mathrm{~mm}$ & 0.43 & 0.43 & 1.03 & 1.08 \\
$-3 \mathrm{~dB} / 12 \mathrm{~mm}$ & 0.96 & 0.79 & 1.85 & 1.81 \\
$-6 \mathrm{~dB} / 12 \mathrm{~mm}$ & 0.47 & 0.46 & 1.27 & 1.11 \\
$+6 \mathrm{~dB} / 8 \mathrm{~mm}$ & 1.03 & 1.05 & 2.97 & 2.51 \\
$+6 \mathrm{~dB} / 5 \mathrm{~mm}$ & 1.18 & 1.23 & 2.84 & 2.61 \\
$+6 \mathrm{~dB} / 3 \mathrm{~mm}$ & 1.15 & 1.14 & 2.91 & 2.71 \\
\hline \hline
\end{tabular}

${ }^{\mathrm{a}} \mathrm{CP}=$ conventional pulsing, $\mathrm{REC}=$ resolution enhancement compression, $\mathrm{FC}=$ frequency compounding, eREC-fC $=$ enhanced REC-FC

with eREC-FC were similar to the results obtained for the REC-FC third-width case regardless of the diameter of the lesion as shown in Table I. Similarly, eREC-FC had better spatial resolution compared to REC-FC third-width case.

Histogram analysis was performed over the same regions used to obtain the estimates of CNR. The HO between the target region and the background regions are listed in Table II. HO for the $12 \mathrm{~mm}$ ATS phantom $+6 \mathrm{~dB},+3 \mathrm{~dB},-3 \mathrm{~dB}$ and $6 \mathrm{~dB}$ contrast targets were $14 \%, 13 \%, 13 \%$ and $9 \%$ smaller for eREC-FC than for CP, respectively. These results suggest that the target detectability improved with eREC-FC compared to $\mathrm{CP}$. Interestingly, for the hydrogel cone phantom the $\mathrm{HO}$ reduction was similar although the $\mathrm{CP} \mathrm{HO}$ values were quite smaller. A $15 \%, 10 \%$, and $10 \%$ reduction in $\mathrm{HO}$ between eREC-FC and CP was observed for the $8 \mathrm{~mm}, 5 \mathrm{~mm}$ and $3 \mathrm{~mm}$ in diameter hydrogel contrast targets, respectively. It was also observed that for these smaller targets, near complete

(a)

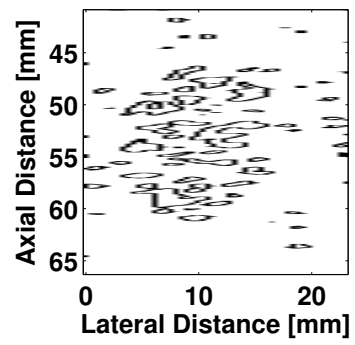

(c)

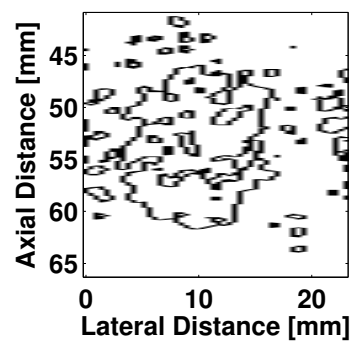

(b)

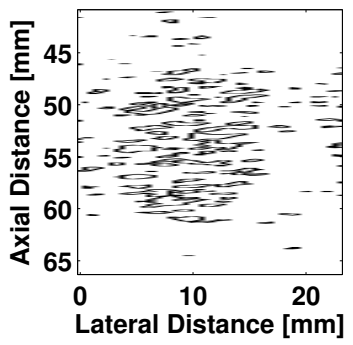

(d)

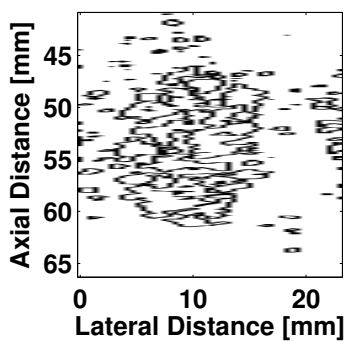

Fig. 7. Margin strength images highlighting the edges in the B-mode images obtained for the ATS phantom $12 \mathrm{~mm},+6 \mathrm{~dB}$ contrast target for (a) $\mathrm{CP}$ (reference), (b) REC (reference), (c) REC-FC (third-width), and (d) eRECFC
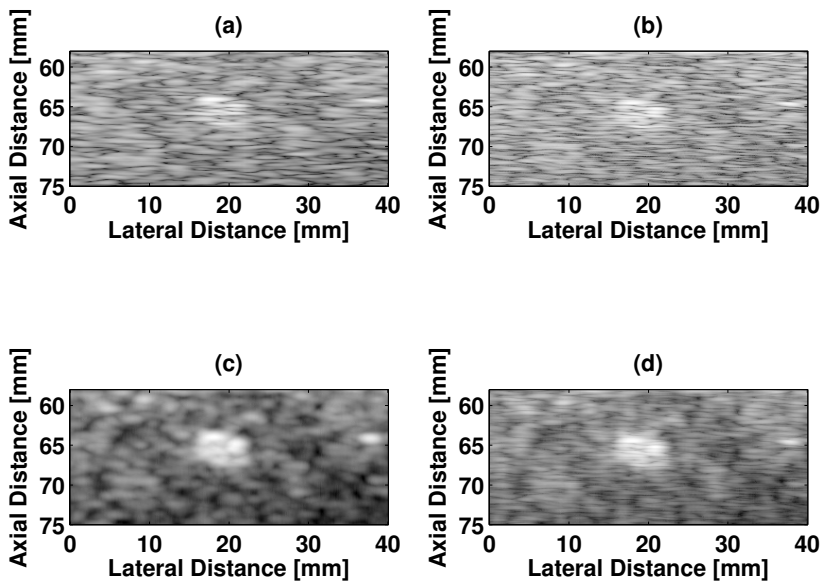

Fig. 8. B-mode images for the hydrogel cone phantom $5 \mathrm{~mm},+6 \mathrm{~dB}$ contrast target for (a) CP (reference), (b) REC (reference), (c) REC-FC (third-width), and (d) eREC-FC

separation between the two histogram regions, target and background was achieved. This suggests that eREC-FC is a useful technique to enhance the detectability of small targets in ultrasonic imaging.

\section{CONCLUSION}

A technique that improves contrast in ultrasound images, known as eREC-FC, was proposed. It was observed that with eREC-FC the quality of the B-mode images generated from echoes of tissue-mimicking phantoms were drastically improved by increasing the CNR. The CNR values obtained with eREC-FC were observed to be within the CNR values estimated for the half width and third-width REC-FC cases. Furthermore, by averaging the CNR of all the REC-FC cases 
TABLE II

HO FOR VARIOUS CONTRAST TARGET FOR CP, REC, REC-FC (THIRD-WIDTH) AND EREC-FC.

\begin{tabular}{c|cccc}
\hline \hline & \multicolumn{4}{|c}{ Technique $^{\mathrm{a}}$} \\
\cline { 2 - 5 } Lesion contrast/diameter & CP & REC & REC-FC & eREC-FC \\
\hline \hline$+6 \mathrm{~dB} / 12 \mathrm{~mm}$ & $36 \%$ & $37 \%$ & $17 \%$ & $22 \%$ \\
$+3 \mathrm{~dB} / 12 \mathrm{~mm}$ & $34 \%$ & $35 \%$ & $21 \%$ & $21 \%$ \\
$-3 \mathrm{~dB} / 12 \mathrm{~mm}$ & $23 \%$ & $25 \%$ & $7 \%$ & $10 \%$ \\
$-6 \mathrm{~dB} / 12 \mathrm{~mm}$ & $27 \%$ & $28 \%$ & $16 \%$ & $18 \%$ \\
$+6 \mathrm{~dB} / 8 \mathrm{~mm}$ & $15 \%$ & $15 \%$ & $1 \%$ & $0 \%$ \\
$+6 \mathrm{~dB} / 5 \mathrm{~mm}$ & $12 \%$ & $11 \%$ & $3 \%$ & $2 \%$ \\
$+6 \mathrm{~dB} / 3 \mathrm{~mm}$ & $11 \%$ & $12 \%$ & $0 \%$ & $1 \%$ \\
\hline \hline
\end{tabular}

${ }^{\mathrm{a}} \mathrm{CP}=$ conventional pulsing, $\mathrm{REC}=$ resolution enhancement compression, $\mathrm{FC}=$ frequency compounding, eREC-fC $=$ enhanced RECFC

used to generate the eREC-FC image also resulted in a $\mathrm{CNR}$ value in between the half width and third-width REC-FC cases. This would suggest that an approximation of the contrast improvements obtained with eREC-FC can be established by averaging the CNR of the images being compounded. Furthermore, the contrast improvements obtained with eRECFC were achieved without deteriorating the axial resolution beyond CP levels.

A potential detriment to eREC-FC technique would be if the difference in contrast between the background and the target is larger than $20 \mathrm{~dB}$. As shown in Fig. 4 the axial resolution at $-20 \mathrm{~dB}$ is double of that for $\mathrm{CP}$. Therefore, a smearing in the image, similar to that observed in the REC-FC study, is possible under targets with large contrast difference with the background (i.e., cystic targets). A potential solution would be to evaluate the image using a sliding window by applying an adaptive filter that preserves brightness at the edges (i.e., keep the original pixel in the image) and smooths the original image otherwise (i.e., replace original pixel in the image by the pixel obtained with eREC-FC technique). The potential tradeoff with this solution could be that small targets, depending on the size of the sliding window, may not be improved using the adaptive eREC-FC technique. Finally, although eREC-FC improved the contrast of ultrasonic Bmode images, further improvements could be obtained by applying several post-processing speckle reduction schemes. These techniques include median filtering, geometric filtering, homomorphic deconvolution, anisotropic diffusion and wavelet filtering.

\section{ACKNOWLEDGMENT}

The authors would like to thank Dr. Roberto J. Lavarello, Professor Michael F. Insana, and Professor William D. OBrien, Jr. from the University of Illinois at Urbana-Champaign for their useful discussions. This work was supported by a grant from the National Institutes of Health (R21 EB006741).

\section{REFERENCES}

[1] C. Burckhardt, "Speckle in ultrasound B-Mode scans," IEEE Trans. Sonics Ultrason., vol. SU-25, pp. 1-6, Jan. 1978.
[2] M. S. Patterson and F. S. Foster, "The improvement and quantitative assessment of B-mode images produced by an annular array/cone hybrid," Utrason. Imag., vol. 5, pp. 195-213, 1983.

[3] J. R. Sanchez an M. L. Oelze, "An ultrasonic imaging speckle-suppression and contrast-enhancement technique by means of frequency compounding and coded excitation," IEEE Trans. Ultrason. Ferroelectr. Freq. Contr., vol. 56, pp. 1327-1339, Jul. 2009

[4] M. L. Oelze, "Bandwidth and resolution enhancement through pulse compression," IEEE Trans. Ultrason. Ferroelectr. Freq. Control, vol. 54, pp. 768-781, Apr. 2007.

[5] J. G. Abbott and F. L. Thurstone, "Acoustic speckle: Theory and experimental analysis," Ultrason. Imaging, vol. 1, pp. 303-324, Oct. 1979.

[6] T. Misaridis and J. A. Jensen, "Use of modulated excitation signals in medical ultrasound. Part I: Basic concepts and expected benefits," IEEE Trans. Ultrason. Ferroelectr. Freq. Contr., vol. 52, pp. 177-191, Feb. 2005.

[7] J. K. Tsou, J. Liu, and M. F. Insana, "Modeling and phantom studies of ultrasonic wall shear rate measurements using coded excitation, IEEE Trans. Ultrason. Ferroelectr. Freq. Contr., vol. 53, pp. 724734, Apr. 2006.

[8] P. M. Shankar, V. A. Dumane, C. W. Piccoli, J. M. Reid, F. Forsberg, and B. B. Goldberg, "Computer-aided classification of breast masses in ultrasonic B-scans using a multiparameter approach," IEEE Trans. Ultrason. Ferroelectr. Freq. Contr., vol. 50, no. 8, pp. 1002-1009, Aug. 2003. 